\title{
EL MOVIMIENTO OBRERO, EL PROTECCIONISMO Y LA LEGISLACIÓN LABORAL. EFECTOS EN UNA EMPRESA TEXTIL DEL DISTRITO FEDERAL EN LA PRIMERA MITAD DEL SIGLO XX José Galindo*
}

RESUMEN: El autor describe el beligerante movimiento laboral que surgió en la fábrica textil La Magdalena hacia finales del Porfiriato y que continuó durante el primer tercio del siglo XX; muestra el efecto que tuvo sobre su capacidad productiva y cómo los "avances" institucionales en materia laboral, entre el gobierno, empresarios y obreros, no ofrecieron incentivos para la transformación tecnológica de la fábrica, que requirió de mayores tarifas para operar. Esta situación, en conjunto con la entrada de las fibras sintéticas como sustitutos del algodón, obligaron al cierre inminente de más de una fábrica textil porfiriana hacia la segunda mitad del siglo pasado.

\begin{abstract}
Tose
ABSTRACT: The author describes the belligerent labor movement at La Magdalena textile factory from the end of the Porfiriato years until the first third of the twentieth century. He demonstrates its effect on production capacity and how institutional advancements in the workforce involving the government, entrepreneurs, and workers were not incentives to the technological transformation of factories but lead to higher operational costs. This occurrence along with the emergence of synthetic fibers substituting cotton led to the imminent closure of many textile factories from the Porfiriato years until the second half of the past century.
\end{abstract}

PALABRAS CLAVE: Porfiriato, Industria textil, La Magdalena, movimiento obrero, legislación laboral, proteccionismo, México.

KEY WORDS: Porfiriato, Textile Industry, La Magdalena, labor movement, labor legislation, protectionism, Mexico.

\footnotetext{
* Departamento Académico de Economía, ITAM.
} 
JOSÉ GALINDO

RECEPCIÓN: 01 de semptiembre de 2011. APROBACIÓN: 02 de febrero de 2012. 


\section{EL MOVIMIENTO OBRERO, EL PROTECCIONISMO Y LA LEGISLACIÓN LABORAL. EFECTOS EN UNA EMPRESA TEXTIL DEL DISTRITO FEDERAL EN LA PRIMERA MITAD DEL SIGLO XX}

\section{El objetivo de este trabajo es pro-}

fundizar en el desarrollo y los efectos del movimiento obrero que surgió en el primer tercio del siglo XX en la empresa textil La Magdalena Contreras, ubicada en la actual Delegación Magdalena Contreras en la ciudad de México. También se mostrará que, en el mismo período, se dieron avances en materia laboral-como el Contrato-ley de 1912, renovado entre 1925 y 1927-, que dieron nuevas garantías y privilegios a los trabajadores y establecieron aumentos en los niveles generales de protección a la industria. Tanto el fortalecido movimiento obrero y las nuevas regulaciones laborales, como el creciente sistema proteccionista -sobre todo a partir de 1927-, que liberó a la industria textil de la competencia externa, tuvieron un efecto negativo en la inversión y desarrollo tecnológico de las empresas textiles mexicanas en los años subsiguientes, lo que pudo haber sido una de las causas de su cierre en la segunda mitad del siglo XX. ${ }^{1}$

${ }^{1}$ Esto fue una característica común en varios sectores económicos de México y América Latina en el período conocido como de "sustitución de importaciones", que tuvo lugar entre mediados de los años treinta y setenta del siglo XX. 
JOSÉ GALINDO

\section{La fábrica La Magdalena}

Los orígenes de la fábrica textil La Magdalena Contreras se encuentran en tiempos coloniales, cuando un sastre de nombre Jerónimo de León construyó un batán ${ }^{2}$ para la producción de telas de lana en 1543, en un lugar llamado Apantepepuzaco, en donde más tarde se estableció el pueblo de Contreras. A partir de ese momento, la propiedad del batán cambió constantemente y fue transformado primeramente en un obraje (centro de producción textil pre-industrial) en 1636, para aumentar la producción de telas. Este obraje no experimentó importantes cambios, sino hasta la década de los años treinta del siglo XIX. Durante esos años se decidió construir una fábrica textil para la producción de hilados y tejidos de algodón. Esta fábrica de nuevo experimentó una serie de cambios de propietario, en parte por los problemas financieros en los que se vio envuelta. ${ }^{3}$ Sin embargo, en 1898 fue adquirida por empresarios franceses y, nunca más, hasta su cierre en 1973, dejó de estar en sus manos.

Es importante mencionar que a menos de un mes de haber sido adquirida por la empresa francesa Meyran Donnadieu y Cia., un incendio destruyó la fabrica textil. ${ }^{4}$ Este incendio parece haber sido intencional y representó una gran ventaja para los dueños de la empresa, ya que una vez cobrado el seguro, La Magdalena dejó de operar con vapor y agua, tecnología que se empezaba a tornar obsoleta, y comenzó a trabajar con energía hidroeléctrica. La siguiente tabla nos permite observar que entre 1893 y 1912, las principales fábricas textiles cambian de fuente de energía para operar y, en general, hay un crecimiento en el tamaño de estas empresas:

${ }^{2}$ Un batán es una rueda giratoria que se mueve por medio de una corriente de agua y que se utiliza para apretar paños de lana. Richard Salvucci, Textiles y Capitalismo en México: Una Historia Económica de los Obrajes, 1539-1840, 1992, México, Alianza, p. 83.

${ }^{3}$ Para un estudio detallado de la empresa textil La Magdalena Contreras, ver Mario Trujillo, "La Fábrica La Magdalena Contreras (1836-1910)" en Carlos Marichal y Mario Cerutti (comps.), Historia de las Grandes Empresas en México 1850-1930, 1997, México, Fondo de Cultura Económica y Universidad Autónoma de Nuevo León, pp. 245-74.

${ }^{4}$ Periódico El imparcial, México, 18 de marzo de 1898. 
TABLA 1

Mayores fábricas textiles de México, 1893-1912

\begin{tabular}{llrlrrrr}
\hline \multicolumn{1}{c}{ Fábrica } & Estado & Año* & Energía & Husos & Telares & Trabajadores & Algodón (kgs) \\
\hline $\mathbf{1 8 9 3}$ & & & & & & & \\
Río Blanco & Ver. & 1892 & Agua y Vapor & 34,000 & 1,000 & 1,220 & $1,200,000$ \\
La Estrella & Coah. & 1868 & Agua y Vapor & 12,000 & 350 & 600 & 500,000 \\
Sn Antonio & D.F & 1883 & Vapor & 11,940 & 350 & 600 & $1,000,000$ \\
Sn Lorenzo & Ver. & 1883 & Agua y Vapor & 11,088 & 300 & 470 & 207,000 \\
Cocolapan & Ver. & 1838 & Agua & 9,840 & 200 & 450 & 276,000 \\
Hércules, Purísima, & & & & & & & \\
S. Antonio & Qro. & 1843 & Agua y Vapor & 9,000 & 650 & 1,080 & $1,200,000$ \\
Barrón and Colmen & Mex. & 1879 & Agua y Vapor & 8,600 & 400 & 500 & 400,000 \\
Miraflores & Mex. & 1840 & Agua y Vapor & 8,436 & 320 & 350 & 650,000 \\
Patriotismo & Pue. & 1879 & Agua y Vapor & 8,000 & 235 & 460 & 460,000 \\
La Magdalena & D.F. & 1836 & Agua y Vapor & 7,768 & 304 & 320 & 400,000 \\
$\mathbf{1 9 1 2}$ & & & & & & & \\
Río Blanco & Ver. & 1892 & Hidroeléctrica & 42,568 & 1,685 & 2,575 & $1,713,956$ \\
Santa Rosa & Ver. & 1898 & Hidroeléctrica & 40,184 & 1,410 & 1,560 & $1,353,664$ \\
Metepec & Pue. & 1902 & Hidroeléctrica & 34,472 & 1,565 & 1,368 & 931,770 \\
La Hormiga & D.F. & 1879 & Hidroeléctrica & 23,300 & 650 & 1,000 & 762,264 \\
La Estrella & Coah. & 1868 & - & 22,448 & 800 & 800 & 74,104 \\
Hércules & Qro. & 1843 & - - & 21,958 & 433 & 400 & 323,064 \\
Cocolapan & Ver. & 1838 & Hidroeléctrica & 20,048 & 872 & 602 & 512,398 \\
Sn Lorenzo & Ver. & 1883 & Hidroeléctrica & 19,048 & 1,036 & 642 & 762,066 \\
Río Grande & Jal. & 1896 & Hidroeléctrica & 18,960 & 742 & 600 & 642,088 \\
La Magdalena & D.F. & 1836 & Hidroeléctrica & 17,128 & 1,767 & 1,000 & 852,706 \\
\hline & & & & & & &
\end{tabular}

Fuente: Aurora Gómez Galvarriato, "The Impact of Revolution: Business and Labor in the Mexican Textile Industry, Orizaba Veracruz 1900-1930”, 1999, PhD. Diss, Harvard University, p. 76.

* Representa la fecha más temprana en la que se encontró información sobre la fábrica.

En el caso particular de la fábrica de Contreras, el cuadro nos muestra que, durante esos años, sus telares aumentaron de 304 a 1,767 y sus husos de 7,768 a 17,128. También su fuerza de trabajo creció, pasando de 320 a 1000 obreros; además, podemos observar que las inversiones de capital hechas a La Magdalena, le permitieron mantenerse entre las diez primeras del país en términos de producción, a pesar de la inauguración de fábricas tan importantes como Santa Rosa y Metepec; y dentro del Distrito Federal, para 1912, esta fábrica mantenía la segunda posición en términos de husos y ganancias esperadas: 
JOSÉ GALINDO

TABLA 2

Husos y ganancias esperadas en las fábricas textiles, Distrito Federal (1912)

\begin{tabular}{lcc}
\hline \multicolumn{1}{c}{ Fábrica } & Husos & $\begin{array}{c}\text { Ganancias Esperadas } \\
\text { (ene-junio 1912) }\end{array}$ \\
\hline La Hormiga & 23,300 & $611,623.64$ \\
La Magdalena & 17,128 & $736,946.17$ \\
La Carolina & 13,784 & $820,304.82$ \\
San Antonio & 12,660 & $262,206.09$ \\
Guadalupe & 6,692 & $179,189.44$ \\
La Perfeccionada & 5,208 & $309,472.82$ \\
La Fama Montañesa & 5,164 & $5,508.70$ \\
El Salvador & 3,664 & $183,091.05$ \\
La Abeja & 2,720 & $198,396.80$ \\
La India & 720 & $6,588.00$ \\
La Corona & - & - \\
La Industrial & - & - \\
\hline
\end{tabular}

Fuente: AGN, México, Departamento del Trabajo, Caja 68, Exp. 8 y Caja 65, Exp. 5.

\section{El movimiento obrero}

A pesar de la importancia de la fábrica de Contreras en la producción de textiles de algodón a nivel nacional, esta fábrica enfrentó un movimiento obrero radical desde finales del siglo XIX. Sin embargo, sobre este tema es difícil determinar hasta qué punto las ideologías anarquista y anarco-sindicalista, ${ }^{5}$ presentes en el movimiento laboral radical en otras regiones del país, influyeron en las empresas textiles ubicadas en el Distrito Federal durante el Porfiriato. En un análisis a nivel nacional, John Hart considera que, para el año de 1880, los anarquistas eran el grupo más poderoso dentro del movimiento laboral mexicano, con más de 50,000 afiliados en el Congreso General Obrero de la Repú-

${ }^{5}$ El anarco-sindicalismo se diferencia del anarquismo en que la primera era una ideología anticapitalista mucho más militante. Sus seguidores utilizaban medios como huelgas generales y el control directo sobre las fábricas. En teoría, los trabajadores organizados en sindicatos adquirirían suficiente poder para controlar los medios de producción, utilizando lo que ellos llamaban "la acción directa". 
blica Mexicana. ${ }^{6}$ Hart argumenta que, antes de 1870 , cuando los trabajadores estaban generalmente organizados en sociedades de ayuda mutua,${ }^{7}$ estas organizaciones pudieron hacer poco por modificar la influencia de los trabajadores sobre los contratos laborales y las políticas salariales, por lo que fueron un blanco para la propagación de ideas radicales. Además, este autor menciona que la difusión de estas ideas se acentuó por las malas condiciones laborales en las fábricas textiles, el subdesarrollo y falta de higiene en los vecindarios, la jornada laboral, que fluctuaba entre 12 y 18 horas diarias, y los bajos salarios. ${ }^{8}$ Sin embargo, la visión de Hart ha sido considerada un tanto exagerada por otros autores. Por ejemplo, John Lear menciona que "aunque las sociedades de ayuda mutua en ocasiones le dieron techo a anarquistas y socialistas $[\ldots]$ el idioma mutualista dominante [...] hablaba no sólo en favor de la unidad entre los trabajadores sino también de la unidad entre los trabajadores y sus superiores". 9

Además, el régimen de Díaz usó la represión desde muy temprano para desmotivar a las organizaciones de trabajadores radicales, y los trabajadores inspirados en ideas anarquistas, quienes rechazaban relacionarse con el gobierno, fueron marginados por medio de la prisión, el reclutamiento militar y otras medidas similares. ${ }^{10}$ Una vez que la amenaza laboral disminuyó en los años 1880, el apoyo del gobierno a las sociedades de ayuda mutua se debilitó. De acuerdo con Rodney Anderson, cuando la militancia laboral resurgió después de $1905^{11}$ hubo

${ }^{6}$ Esta organización obrera fue fundada en el año de 1876, y de acuerdo con Hart, líderes anarquistas la dirigían. John Hart, El Anarquismo y la Clase Obrera Mexicana, 1860-1930, 1980, México, Siglo XXI, p. 79.

${ }^{7}$ Las sociedades de ayuda mutua eran organizaciones voluntarias en donde los trabajadores hacían contribuciones monetarias periódicas para crear fondos de ahorro para fines de salud y seguro de vida.

${ }^{8}$ Hart, op. cit., p. 60.

${ }^{9}$ John Lear, Workers, Neighbors and Citizens. The Revolution in Mexico City, 2001, Lincoln, University of Nebraska Press, p. 111.

${ }^{10}$ Ibid., p. 114.

${ }^{11}$ Anderson argumenta que el origen del enojo de los trabajadores mexicanos en ese tiempo tenía razones obvias: estaban mal pagados, trabajaban en exceso, eran maltratados y, en ocasiones, discriminados en contra de trabajadores extranjeros. Rodney Anderson, Outcasts in their own Land. Mexican Industrial Workers, 1906-1911, 1976, Dekalb, Northern Illinois University Press, p. 311. 
JOSÉ GALINDO

un cambio en la política laboral de nuevo. Parecía, según Anderson, que Díaz tenía un deseo, probablemente motivado por razones políticas, de resolver cierto número de demandas laborales. Por ejemplo, después de la revuelta textil de Río Blanco en 1907, Díaz realizó esfuerzos para iniciar una reforma en las regulaciones de las fábricas en la región de Orizaba. Por otro lado, continuó utilizando la represión en contra de otros grupos de trabajadores. Por ejemplo, en contra de trabajadores que apoyaban a Madero, quien en ese tiempo era líder del "Partido Antireeleccionista" de oposición. ${ }^{12}$ Aunque Díaz probablemente tenía un deseo genuino de hacer algunos cambios en las condiciones laborales de los obreros, no estaba dispuesto a ponerse en contra de los empresarios, quienes tenían un peso fundamental en el sector económico y financiero de México. Además, su gobierno carecía de una política laboral consistente y de un mandato legal para hacer frente a las demandas laborales. ${ }^{13}$ En 1910, la campaña electoral de Madero obtuvo un fuerte apoyo de los obreros urbanos. ${ }^{14}$ Anderson menciona que la mayoría de estos trabajadores se identificaban con Madero y su ideología liberal más que con ideologías revolucionarias como el anarquismo o el programa radical de los hermanos Flores Magón. ${ }^{15}$ Sin embargo, aunque el autor considera al anarquismo como la hipótesis más débil para explicar la motivación de los trabajadores para organizarse, no descarta la posible influencia de esta ideología. ${ }^{16}$ John Hart señala a la fábrica La Magdalena como "un sitio de militancia laboral anarquista [...] entre 1876-1882 y 1911-1931 y huelgas continuas en los

${ }^{12}$ Ibid., p. 301.

${ }^{13}$ Ibid., pp. 301-2.

${ }^{14}$ Ver Coralia Gutiérrez, Experiencias Contrastadas: Industrialización y Conflictos en los Textiles del Centro-Oriente de México, 1884-1917, 2000, México, El Colegio de México y BUAP, pp. 220-3.

${ }^{15}$ Los hermanos Flores Magón eran los líderes del Partido liberal mexicano, que era un partido de oposición que, en un inicio, "propuso el retorno a los principios de las facciones radicales del movimiento liberal al mando de Juárez. (Pero después), el aumento de la represión gubernamental contribuyó a un rápido giro a la izquierda, y el partido pronto adoptó el camino y los pronunciamientos anarco-sindicalistas". Friedrich Katz, en Leslie Bethell (ed.), Mexico Since Independence, 1991, Cambridge, Cambridge University Press, p. 112.

${ }^{16}$ Anderson, op. cit., p. 313. 
1880s y 1890 s". ${ }^{17}$ Sin embargo, Hart no muestra evidencia contundente sobre la afirmación anterior, por lo que es probable que su obra confunda militancia anarquista con militancia laboral que, en efecto, fue constante en esta fábrica. ${ }^{18}$ De hecho, entre 1906 y 1911, diez de las once huelgas textiles que surgieron en fábricas textiles del algodón brotaron en La Magdalena, La Hormiga y San Antonio Abad. ${ }^{19}$ Hacia finales del Porfiriato, la clase trabajadora, en general, había fallado en crear sindicatos fuertes y durables y en lograr que sus demandas fueran resueltas. Además, los trabajadores textiles no gozaban de la protección de contratos escritos, leyes laborales favorables y sindicatos nacionales de peso. ${ }^{20}$ En el Distrito Federal hubo algunos esfuerzos por parte del gobernador Guillermo Landa y Escandón de crear la Sociedad Mutualista y Moralizadora de Trabajadores del Distrito Federal a fines de 1909. ${ }^{21}$ Sin embargo, sus esfuerzos fracasaron, ya que el régimen Porfiriano cayó poco tiempo después.

Esta mala situación de los trabajadores cambiaría durante la Revolución. En diciembre de 1911, a menos de dos meses de que Madero llegara a la presidencia, una huelga textil importante comenzó en Puebla. Los trabajadores de muchas fábricas poblanas iniciaron la huelga

${ }^{17}$ Hart, op. cit., p. 99.

${ }^{18}$ Es un hecho que una de las críticas al trabajo de Hart, y a algunos otros que hablan sobre la influencia del anarquismo en México, es su falta de evidencia sobre los vínculos directos entre los anarquistas y las fábricas. En este sentido, el libro de John Lear, antes citado, es una buena referencia. Lo que es verdad es que La Magdalena sí fue una fábrica víctima de militancia laboral. Por dar dos ejemplos en un período temprano: en 1878, La Magdalena sufrió una huelga de un mes aproximadamente, en demanda de menos horas de trabajo. Sin embargo, el intento por resolver este problema no fue exitoso y algunos de los huelguistas fueron despedidos y tuvieron que buscar trabajo en haciendas cercanas. Ver Moisés González, Las Huelgas Textiles en el Porfiriato, 1970, México, José M. Cajica, p. 24. En 1885, los trabajadores de la fábrica de Contreras se rebelan para prevenir una reducción de salario del $50 \%$, pero los dueños buscaron la manera de despedir a estos obreros y contrataron a nuevos con muy bajos salarios. Hay muchos otros ejemplos en donde los obreros de La Magdalena se rebelan en los 1870 y 1880. Una buena referencia es el libro citado de Moisés González.

${ }^{19}$ Anderson, op. cit., p. 306.

${ }^{20}$ Jeffrey Bortz, "Without any more Law than their own Caprice: Cotton Textile Workers and the Challenge to Factory Authority During the Mexican Revolution", International Review of Social History, 1997, 42: 253-288, p. 263.

${ }^{21}$ Esta sociedad incluiría representantes de los trabajadores, empresarios y el gobierno para resolver conflictos laborales en la región. 
JOSÉ GALINDO

y fueron seguidas por fábricas de Tlaxcala y el Distrito Federal, incluyendo a La Magdalena. Las demandas más comunes eran sobre salarios y jornada laboral. ${ }^{22}$ Esta huelga definiría la política laboral que la administración de Madero seguiría.

En julio de 1912, el gobierno convocó a la primera Convención de Industriales Textiles. En ella se reunieron representantes del sector industrial y obrero y también contó con la presencia de representantes del gobierno. La Magdalena envió a dos representantes, Tomás Reyes Retana y Luis Veyan. ${ }^{23}$ El resultado de la reunión fue el "Reglamento de Hilados y Tejidos de la República”, un contrato laboral aprobado por los representantes de los tres sectores que intervinieron y publicado en el mismo mes por la Secretaría de Desarrollo. Este fue el primero de los contratos laborales que aparecería en la industria textil en el siglo XX. El nuevo reglamento tocó temas como la jornada laboral, multas a los obreros por daños causados al material de trabajo o trabajos defectuosos y comisiones mixtas para resolver problemas entre obreros y patrones. Además, los industriales también aprobaron la "Tarifa de Hilados y Tejidos", una nueva escala de salarios que garantizaba un salario mínimo de un peso por día para los obreros textiles. ${ }^{24}$ Este fue un importante paso hacia la institucionalización de la participación del gobierno en el incipiente sistema de relaciones laborales. Hasta ese entonces, las negociaciones contractuales habían sido principalmente una cuestión privada. $^{25}$

Después de la Convención, los obreros se empezaron a sentir lo suficientemente fuertes para limitar la autoridad de los empresarios. Además, entendieron que para el gobierno del momento se volvió más difícil reprimirlos y, por lo mismo, podían ganar batallas en contra de los empresarios. Bortz muestra cómo en diferentes fábricas, los obreros empezaron a ser gobernados "sin ninguna ley más que su propio

${ }^{22}$ Coralia Gutiérrez, op. cit., pp. 332-3.

${ }^{23}$ AGN, México, DT, Caja 16, Exp. 14, ES. $2,3$.

${ }^{24}$ Sesión, 29 de julio 1912, AGN, México, DT, Caja 15, Exp. 18; y Periódico El Imparcial, México, 2 de agosto de 1912.

${ }^{25}$ Jeffrey Bortz, "The Genesis of the Mexican Labor Relations System: Federal Labor Policy and the Textile Industry 1925-1940”, The Americas, 1995, 52(1): 43-69, p. 47. 
capricho", ${ }^{26}$ El gobierno también empezó a jugar un papel importante en estos cambios mediante el nuevo Departamento del Trabajo, inaugurado a principios de 1912, que supervisaba el cumplimiento de la nueva regulación laboral en las fábricas. Esta práctica continuó hasta después de la caída de Madero. Por ejemplo, en diciembre de 1913 Miguel Casas, inspector del Departamento del Trabajo, visitó La Magdalena para supervisar si la fábrica estaba cumpliendo con la regulación y los salarios aprobados el año anterior. ${ }^{27}$

A pesar de que estos cambios estaban llevándose a cabo, la capacidad de los trabajadores para limitar el control de los dueños en las fábricas no debe ser exagerada. En muchas ocasiones, los dueños no pagaron los salarios acordados en la Convención de 1912. Por ejemplo, en marzo de 1920, José Pérez, representante del sindicato de La Magdalena, solicitó al Departamento del Trabajo que enviara a un inspector porque "los salarios en el departamento de hilados estaban lejos de ser los acordados en la convención de $1912 "{ }^{28}$ Lo que es un hecho es que la simple posibilidad de solicitar una petición de este tipo a una institución gubernamental representaba un claro avance en la institucionalización de las relaciones obrero-patronales, con el gobierno jugando el papel de árbitro final, que no retrocedería.

Después de la huelga general de diciembre de 1911, radicales en la Ciudad de México se organizaron en la Casa del Obrero Mundial. La Casa era una asociación anarco-sindicalista que obtuvo apoyo de organizaciones de trabajadores a las que la administración de Madero no había dado participación política directa ni mejoramientos significativos en sus lugares de trabajo. Sin embargo, de acuerdo a Lear, no fue la ideología anarco-sindicalista lo que movió a los trabajadores a afiliarse a la Casa, sino su deseo de "eliminar los peores abusos en la industria $[\ldots]$ garantizando salarios y condiciones de trabajo justas". ${ }^{29}$

${ }^{26}$ Frase que aparece en una carta escrita por el gerente de la fábrica de San Ildefonso al Departamento del Trabajo, citada por Jeffrey Bortz, "Without any more Law than their own Caprice, op. cit., p. 268.

${ }^{27}$ AGN, México, DT, Caja 32 Ex. 11.

${ }^{28}$ AGN, México, DT, Caja 211 Ex. 21.

${ }^{29}$ Lear, op. cit., p. 361. 
JOSÉ GALINDO

La Casa pronto empezó a obtener poder dentro del movimiento obrero del Distrito Federal. Al menos en el México central, esta organización atrajo a más seguidores que los sindicatos oficiales como la Gran Liga Obrera de la República Mexicana creada por la administración de Madero.

A pesar de que la Casa fue cerrada por la facción constitucionalista de la Revolución después de que esta facción abatió violentamente una huelga general que emergió en la Ciudad de México, esto no dañó al movimiento obrero independiente, como autores como Ramón Ruiz han afirmado. ${ }^{30}$ Bortz y Lear coinciden en que, a pesar de los problemas que llevaron a la caída de la Casa, la organización obrera independiente se mantuvo durante la Revolución. ${ }^{31}$ De hecho, Cándido Aguilar, gobernador de Veracruz, consciente de la fuerza que adquiría día con día la clase obrera independiente, emitió un decreto en diciembre de 1916 que daba varios beneficios a los trabajadores de su estado. Por otro lado, la Constitución de 1917 también incluía varias de las peticiones que los obreros habían buscado en la década previa. Por ejemplo, el artículo 123 daba al trabajo y al capital el derecho a organizarse en función de sus respectivos intereses, permitía a los trabajadores negociar colectivamente y establecía el derecho de huelga. ${ }^{32}$

Para ese entonces, el fortalecido movimiento obrero continuó organizando huelgas, en algunos casos sin causa justa. Por ejemplo, a principios de 1920, la fábrica de Santa Teresa, que estaba ubicada a un costado de La Magdalena y pertenecía a los mismos dueños franceses, tuvo una huelga de tres días que, básicamente, apoyaba a un trabajador que había sido despedido por una causa justa. ${ }^{33}$ Además, los trabajado-

${ }^{30}$ De acuerdo con Ruiz, "para fines de 1916 los gobernantes de México habían dado un golpe mortal a la Casa y al movimiento obrero independiente". Ramón Ruiz, Labor and Ambivalent Revolutionaries. Mexico 1911-1923, 1976, Baltimore, John Hopkins University Press, p. 293.

${ }^{31}$ Lear, op. cit., p. 364, y Bortz, 1997, op. cit., p. 270.

${ }^{32}$ El inicio de la aplicación del artículo 123 de la Constitución de 1917 tardó tiempo, ya que los industriales se rebelaron fuertemente y "Carranza no hizo intentos serios por reglamentar el artículo [...] y se mostró demasiado condescendiente con los industriales". Ver Enrique Guerra, "Proceso de Trabajo y Movimiento Obrero en la Industria Textil Mexicana (1912-1927)", 1992, Tesis de Maestría, México, Flacso, pp. 176-9.

${ }^{33}$ Ver Bortz, op. cit., 1997, p. 280. 
res comenzaban a bloquear la modernización de las fábricas textiles. Por ejemplo, a principios de los 1920 algunos empresarios intentaron invertir en tecnología moderna como telares automáticos en sus fábricas. A pesar de que esta tecnología era común en muchos países industrializados, los sindicatos de las fábricas no permitieron su instalación. En el caso particular de la fábrica textil Atoyac, cuando en 1923 con la aprobación del gobierno ya habían sido instalados nuevos telares automáticos, "los sindicatos impidieron su operación. El trabajador que se encargaba de la operación de estos telares fue asesinado. Su sucesor pronto comenzó a recibir amenazas de muerte y renunció. Nadie más aceptó el manejo de estos telares y fueron abandonados hasta que algunos técnicos los transformaron en telares ordinarios". 34

La militancia laboral continuaría por algunos años. Después de la caída de La Casa del Obrero Mundial, la Confederación Regional Obrera Mexicana (CROM), fundada en 1918, atrajo a algunos de sus ex-miembros. Sin embargo, otros fundaron la Confederación General del Trabajo (CGT) en 1921. Esta organización mantuvo la ideología anarco-sindicalista y fue muy beligerante en sus primeros seis años. Lo interesante de mencionar es que la CGT atrajo a los sindicatos de las fábricas de San Ángel, incluyendo La Magdalena. En 1922 estalló una huelga en San Ildefonso, fábrica textil de lana ubicada en el Estado de México. Como las demandas de los huelguistas no fueron satisfechas, la CGT llamó a una huelga general incluyendo a todas las fábricas textiles del Distrito Federal "confiando en que los trabajadores textiles del D.F. estaban afiliados a esta organización". ${ }^{35}$ Los trabajadores textiles de La Magdalena apoyaron esta huelga, que duró aproximadamente un mes y finalizó en el municipio de San Ángel, donde cerca de 5,000 trabajadores, principalmente de la fábrica Santa Teresa, protestaron en contra del secuestro de un líder sindical textil. Mientras se encontraban en el municipio, la policía montada les disparó. Muchos de ellos fueron heri-

${ }^{34}$ Jesús Rivero Quijano, La Revolución Industrial y la Industria Textil en México, 1990, México, Joaquín Porrúa Editores, 2 vols. p. 278.

${ }^{35}$ Luis Araiza, Historia del Movimiento Obrero Mexicano, 1975, México, Casa del Obrero Mundial, p. 75. 
JOSÉ GALINDO

dos y al menos dos murieron. ${ }^{36}$ Esta huelga tuvo una trascendencia fundamental, ya que después de ella muchos trabajadores se afiliaron a la CGT y los anarco-sindicalistas recuperaron algo de su poder.

La CGT aseguraba tener 80,000 afiliados entre 1928-29, la mayoría de ellos en la Ciudad de México. ${ }^{37}$ Los trabajadores textiles eran una vez más uno de los grupos más importantes atraídos por una organización anarco-sindicalista. Sin embargo, el gobierno estaba claramente en contra de la CGT. Así, los industriales apoyados por el gobierno no hicieron ninguna concesión adicional a los afiliados a la CGT. Por ejemplo, en el Distrito Federal, los empresarios textiles cerraron sus fábricas en algunos períodos entre 1923 y 1924, argumentando que las materias primas eran escasas. Sin embargo, un reporte mostró que algunas fábricas tenían suficiente material para operar por lo menos por tres meses más. Los miembros de la CGT declararon que las plantas debían de continuar abiertas o los trabajadores tomarían control de ellas. Pero el gobierno, representado por el General Manuel Pérez Treviño, apoyó la postura de los empresarios. Esto llevó a varias huelgas en las fábricas. ${ }^{38}$

En 1924, Calles, junto con Morones, líder de la CROM y nuevo Secretario de Industria, empezó a combatir a todos los sindicatos de la CGT. Aunque la CROM tenía una minoría de afiliados en las fábricas textiles del D.F., en 1925 el gobierno apoyó la invasión de las oficinas de la CGT en el área de Contreras por parte de aquella organización laboral. Esto tuvo como consecuencia muchas revueltas violentas en las calles, huelgas, paros patronales e intervenciones de la policía militar en apoyo a la minoría de la CROM. ${ }^{39}$

En ese tiempo, la CROM estaba expandiendo su influencia en todo el país, emitiendo leyes y aumentando su burocracia. Por lo que, debido a la difícil situación de la CGT, los CGTistas comenzaron a ver a la CROM como una alternativa. Esto ayudó a centralizar el control del

${ }^{36}$ Guerra, op. cit., pp. 224-5.

${ }^{37}$ Hart, op. cit., p. 195.

${ }^{38}$ Rosendo Salazar, Historia de las Luchas Proletarias, 1923 a 1936, 1938, México, Avante, 2 vols., pp. 119-25.

${ }^{39}$ Hart, op. cit., p. 204. 
movimiento obrero en manos del gobierno. La CROM aseguraba tener dos millones de afiliados en 1928, pero reconocía que al menos la mitad de estos eran campesinos. Meyer menciona que, en la ausencia de mejor información, "se puede mencionar que la CROM debe haber reunido a 100,000 trabajadores, artesanos, burócratas, pequeños comerciantes, y a 50,000 agricultores". ${ }^{40}$ Sin embargo, la CGT continuaría en la batalla por un tiempo. En julio de 1925, la Junta de Conciliación y Arbitraje (JCA) apoyó una demanda hecha por los dueños de tres de las fábricas ubicadas en la región de Contreras, una de ellas La Magdalena. Los empresarios demandaron un ajuste de salarios. El apoyo de la JCA a los empresarios llevó a varios conflictos con los obreros. Por ejemplo, el gobierno federal culpó a los trabajadores de La Magdalena afiliados a la CGT porque evitaron la entrada de los trabajadores afiliados a la CROM y porque convocaron a una junta ilegal dentro de la fábrica ${ }^{41}$ En noviembre del mismo año, La Magdalena experimentó peleas intensas entre los trabajadores de las dos confederaciones laborales, después de que los dueños contrataron trabajadores de la CROM. Al siguiente año, los dueños de La Magdalena despidieron a 50 trabajadores de la CGT y el gobierno mandó tropas federales para proteger a los trabajadores de la CROM y a la fábrica misma. ${ }^{42}$ Para 1928, la CGT se mantenía poderosa en el Distrito Federal. Sin embargo, el asesinato del presidente electo Álvaro Obregón, que debilitó a la CROM, y la muerte de los líderes más radicales de la CGT, hicieron posible una mejor relación de la CGT con el gobierno en el futuro.

\section{El efecto de la militancia laboral en La Magdalena}

A estas alturas, es importante tratar de analizar el efecto que tuvo la militancia laboral en la fábrica La Magdalena. ${ }^{43}$ Una conclusión obvia

${ }^{40}$ Jean Meyer, en Leslie Bethell, (ed.), Mexico Since Independence, 1991, Cambridge, Cambridge University Press, p. 229.

${ }^{41}$ Salazar, op. cit., p. 196-210.

${ }^{42}$ Ibid., p. 196-210.

${ }^{43}$ Es muy difícil suponer y comparar el nivel de producción e ingresos de esta fábrica con y sin militancia laboral. Sin embargo, en este trabajo se harán unas conclusiones parciales 
JOSÉ GALINDO

es que cualquier empresario textil hubiese preferido no tener trabajadores afiliados a organizaciones radicales. Sin embargo, pareciera que el hecho de haber tenido un movimiento obrero militante, no fue un obstáculo para que La Magdalena se mantuviera dentro de las fábricas que generaban más producto en el Distrito Federal y en el país en general. Hay algunas razones por las que la fábrica pudo mantener una buena posición: en primer lugar, los obreros necesitaban el trabajo. Esto es, en general, gente que provenía de las áreas rurales aledañas en busca de mejorar su condición de vida por lo que debían continuar trabajando. En segundo lugar, existía un importante control sobre ellos en la fábrica: había supervisores que mantenían el control de los trabajadores y su actividad, registrando información sobre su desempeño en tarjetas especiales. Como los supervisores eran empleados de confianza con mejores salarios, no eran fácilmente sobornables. En tercer lugar, aunque los empresarios franceses, propietarios de La Magdalena, enfrentaron un movimiento obrero más dinámico y organizado que sus antecesores, el gobierno rechazó generalmente las ideologías y a las organizaciones radicales y, en ocasiones, ayudó a los industriales a combatirlas. En cuarto lugar, aunque la fábrica enfrentó muchas huelgas y paros patronales, el mercado no demandó el total de la producción de las fábricas. Por ejemplo, en más de una ocasión entre 1923 y 1924, industriales de Puebla, Tlaxcala y la Ciudad de México solicitaron el permiso del gobierno federal para disminuir las horas de trabajo en las fábricas, con el objeto de reducir el exceso de producción acumulada. ${ }^{44}$ Stephen Haber menciona que, no obstante, debido a los datos imprecisos, es imposible estimar de manera precisa las tasas de capacidad utilizada en la industria textil, un fenómeno de sobreproducción ocurría de manera regular "al menos dos veces en la última etapa del Porfiriato (1901-2 y 1907-8); durante los años de la Revolución (1910-17); y en los años 1920 el problema era endémico". ${ }^{5}$

a partir de la posición relativa de La Magdalena con respecto a otras empresas en términos principalmente de niveles de producción.

${ }^{44}$ Bortz, op. cit., p. 48.

${ }^{45}$ Stephen Haber, Industry and Underdevelopment: The Industrialization of México 1890-1940, 1989, Stanford, Stanford University Press, pp. 33-4. 
La siguiente tabla muestra la importante posición que Veyan Jean Compañía y Sucesores (VJCS), propietaria de La Magdalena, mantuvo dentro de los productores textiles del algodón entre 1910 y 1929, año en el cual el movimiento obrero radical, como observamos anteriormente, comenzó a mejorar su relación con el gobierno. ${ }^{46}$ La tabla también muestra cómo otras compañías propietarias de fábricas con un gran componente de afiliados a la CGT, como La Hormiga y San Antonio Abad, mantuvieron igualmente una buena posición.

En la tabla también se pueden observar los efectos negativos que la Revolución pudo haber tenido en los niveles de producción de La Magdalena. Podemos ver que su posición entre las 10 compañías más grandes en la industria textil del algodón pasó del séptimo lugar en 1910 al décimo en 1915. De hecho, existe evidencia de que, entre esos años, La Magdalena enfrentó invasiones zapatistas y escasez de materias primas. ${ }^{47}$ Sin embargo, para 1920 la compañía VJCS había recuperado su posición relativa, ocupando el sexto lugar dentro de los productores nacionales. Durante los años veinte, La Magdalena mantuvo también una posición privilegiada dentro de la industria textil del algodón, produciendo más del tres por ciento de la producción textil total en los años de 1925 y $1929 .{ }^{48}$

${ }^{46}$ Es importante mencionar que, para el año de 1929, la Compañía VJCS solamente contaba con dos fábricas textiles de algodón. Además de La Magdalena, esta compañía era propietaria de la fábrica Río Hondo, ubicada en Naucalpan, Edo. de México. Esta fábrica fue adquirida hasta el año de 1923 y representaba aproximadamente una cuarta parte del tamaño de La Magdalena. Lo que se quiere enfatizar aquí es que en la tabla 3, los datos están basados en la producción de La Magdalena básicamente. Ver Secretaría de Hacienda y Crédito Público, Departamento de Impuestos Especiales, Directorio de las Fábricas de Hilados y Tejidos Registradas, 1938, México.

${ }^{47}$ Eduardo Oropeza y Magdalena Martínez, "Participación de Contreras en la Lucha Zapatista", 1986, México, La Voz de Contreras, 28.

${ }^{48} \mathrm{El}$ contar con información relativa impide la posibilidad de hacer algunas afirmaciones con certidumbre. Sin embargo, con la información que tenemos, sí se puede afirmar que en los años en que la fábrica textil La Magdalena enfrentó el movimiento obrero más radical, se mantuvo dentro de las fábricas que más textiles producían del país. Es muy difícil evaluar qué hubiera sucedido si esta fábrica hubiese tenido obreros menos belicosos, pero lo que es un hecho es que la fábrica no terminó siendo víctima de las acciones de sus trabajadores radicales. Es decir, pudo subsistir manteniéndose con altos niveles de producción. 
JOSÉ GALINDO

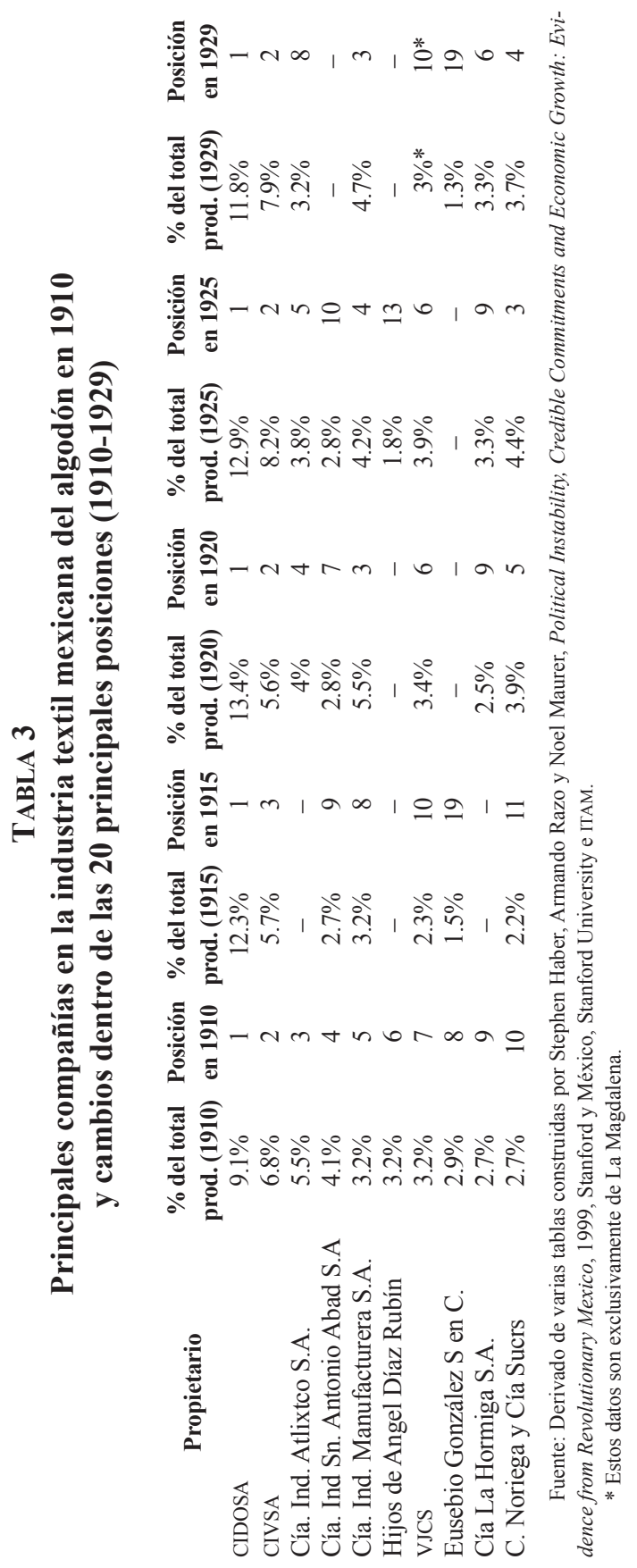




\section{La convención obrero-patronal de 1925-1927}

Los presidentes que emergieron de la Revolución no fueron lo suficientemente fuertes para imponer su voluntad sobre el fortalecido movimiento obrero que había surgido en esos años. La estrategia, entonces, fue la de construir un consenso alrededor de las nuevas instituciones laborales. Sin embargo, en la primera mitad de los años veinte, la industria textil estaba enfrentando problemas que parecían difíciles de resolver: los trabajadores demandaban incrementos salariales en un ambiente económico adverso, donde las fábricas habían sido golpeadas por una caída en la demanda de sus productos. En parte, con el propósito de resolver estos problemas, la administración de Calles convocó a una convención textil tripartita: la Convención Obrero Patronal, similar a la Convención de 1912. Se llevó a cabo entre 1925 y 1927 y participaron los representantes del sector obrero de 110 fábricas y los dueños de $117 .{ }^{49}$ Uno de los dueños de La Magdalena, Camilo Veyan, acudió como uno de los cinco empresarios que representaron las posturas de todos los industriales textiles del país. ${ }^{50}$

En general, las acciones tomadas por el gobierno durante la Convención tenían el propósito de extender la autoridad federal en la cuestión laboral y obtener el apoyo de los trabajadores en elecciones y en levantamientos militares. ${ }^{51}$ Las negociaciones con respecto a los salarios dividieron a los industriales, lo que permitió que el gobierno aumentara su papel en el debate. ${ }^{52}$ Entre otros asuntos, la Convención aprobó la "cláusula de exclusión”, que daba el derecho a los líderes sindicales de expulsar trabajadores por realizar actividades en contra de sus intereses. Esto, sin duda, sirvió para limpiar las fábricas de obreros no deseados ni por los sindicatos ni por el gobierno, fortaleciendo de esta manera a un grupo homogéneo de trabajadores vinculado al Estado. Aunque individualmente los trabajadores no ganaron tanto con esta cláusula, obtuvieron una victoria magistral cuando la Convención aprobó el arbi-

${ }^{49}$ Secretaría de la Economía Nacional, La Industria Textil en México: El Problema Obrero y los problemas Económicos, 1934, México, Talleres Gráficos de la Nación, p. 48.

${ }^{50}$ Bortz, op. cit., p. 48.

${ }^{51}$ Haber, op. cit., Cambridge, Cambridge University Press, p. 149.

${ }^{52}$ Secretaría de la Economía Nacional, La Industria Textil en México, op. cit., pp. 24 y 52. 
JOSÉ GALINDO

traje en asuntos relacionados con los despidos, creando para ello comisiones bilaterales. Mientras que el contrato de 1912, en su artículo 11, mantuvo cierta autonomía por parte de los empresarios para decidir cuándo despedir a algún trabajador, el artículo 81 del contrato de 1927 creó un sistema de "comisiones mixtas" que dificultaba de manera considerable el despido de los trabajadores. Además de esta inflexibilidad introducida en el mercado laboral, que generaría muchos problemas a los industriales, la nueva legislación también introdujo más obstáculos para la inversión y modernización de la industria. ${ }^{53}$ Específicamente, la nueva lista de salarios que fue estipulada fijaba un máximo número de máquinas por trabajador y establecía salarios específicos por pieza producida. Bajo estas condiciones, los empresarios no tenían incentivos para introducción de mejor tecnología, porque no les permitiría reducir costos laborales, ya que los salarios por pieza tenían que mantenerse fijos. ${ }^{54}$ En particular, esta lista impidió la introducción de la maquinaria de punta de los años veinte: los telares de dos tamaños, que aumentaban la productividad de los tejedores; el batiente de un solo proceso, que reducía la recolección y empacado de algodón a un solo proceso; y los altos estirajes, que reducían las veces que el hilo pasaba por los veloces. ${ }^{55}$

Después de la Convención de 1925-27, fue necesario incrementar el nivel de protección para mantener la industria operando. Por ejemplo, aunque la tarifa ad valorem para tela blanca y manta se había incrementado en $61.7 \%$ entre 1911 y 1926, ésta aumentó de $32.5 \%$ en 1926 a $186.3 \%$ en 1927 . En el caso de tela fina blanca y $\sin$ blanquear, la tarifa creció en 44.2\% entre 1911 y 1926, pero entre 1926 y 1927 pasó de 40.8\% a $215 \% .{ }^{56}$ Se puede concluir entonces que en la Convención Obrero patronal de 1925-27 los tres principales actores en la economía política

${ }^{53}$ Ya vimos cómo el poder que habían adquirido los sindicatos en los años veinte impidió la introducción de telares automáticos en varias fábricas.

${ }^{54}$ Secretaría de la Economía Nacional, Monografia Económico Industrial de la Fabricación de Hilados y Tejidos de Algodón, 1933, México, Mimeo, p. 67.

${ }^{55}$ Rivero Quijano, op. cit., pp. 239-80.

${ }^{56}$ Aurora Gómez Galvarriato, "The Political Economy of Protectionism: The evolution of labor Productivity, International Competitiveness, and Tariffs in the Mexican Textile Industry, 1900-1950”, 2001, México, Centro de Investigación y Docencia Económicas, pp.7-10. 
de la industria textil: empresarios, obreros y gobierno, eligieron un arreglo institucional que no ofreció incentivos a la transformación tecnológica $\mathrm{y}$, como consecuencia, requirió de mayores tarifas para operar.

\section{Efectos de los cambios, después de los años veinte}

Esta es una breve sección en la que se busca dejar ver cómo los efectos de los cambios en la legislación laboral en la fuerza adquirida por los sindicatos y en el aumento del proteccionismo se resintieron en años posteriores a 1920 .

La situación de la industria textil del algodón cambió durante los años treinta. Entre 1932 y 1937 la producción creció en más 60\% y esto fue acompañado de un aumento en la productividad del trabajo y capital. ${ }^{57}$ Para 1937, el Departamento del Trabajo decidió convocar a una nueva convención revisora de la de la década anterior: la "Convención Obrero Patronal Revisora de la Convención Colectiva del Trabajo y Tarifas Mínimas (1925-27) en el Ramo de Algodón”. Como en la convención anterior, los empresarios estaban divididos entre la Mayoría (dueños principalmente de fábricas textiles de Puebla y Tlaxcala) y la Minoría (dueños principalmente de fábricas textiles de la Ciudad y el Estado de México y Veracruz), como se les conocía. Los representantes del trabajo también estaban divididos entre la CTM, CGT y dos facciones de la CROM. Fue tal el debate sobre la rigidez de la lista de salarios, que el asunto tuvo que ser tornado al presidente Cárdenas, quien contribuyó para que se estableciera un nuevo contratoley laboral en abril de 1939.

De alguna manera, las resoluciones de la Convención afianzaron el sistema creado diez años antes. Los trabajadores continuaban rechazando la innovación tecnológica para poder conservar sus plazas, por lo que el gobierno mantuvo la inflexibilidad de la lista de salarios que había sido acordada en la convención de 1925-27. ${ }^{58}$ Por otro lado, las

${ }^{57}$ Haber, op. cit., p. 180.

${ }^{58}$ Secretaría de Hacienda y Crédito Público, Comisión de Aranceles, La Industria Textil del Algodón en México, 1940, México, Mimeo, pp. 19-23. 
JOSÉ GALINDO

tarifas se mantuvieron lo suficientemente altas, protegiendo así a los empresarios de la competencia externa. ${ }^{59}$ Además, los salarios acordados en el nuevo contrato fueron mantenidos bajos, permitiendo a los empresarios enfrentar menores costos. ${ }^{60}$

El período que comprende la Segunda guerra mundial fue el último en que las fabricas textiles que habían aparecido desde mediados del siglo XIX en México tendrían altos volúmenes de producción. ${ }^{61}$ Las exportaciones de textiles de algodón representaron el 60 por ciento del total de las exportaciones manufactureras durante la guerra. En esos años, las fábricas textiles operaban en tres turnos de ocho horas diarias para satisfacer la demanda estadounidense fundamentalmente. ${ }^{62}$ En el caso de la fábrica La Magdalena, "entre los años 1940-1944 trabajaba día y noche y sus productos se enviaban a Francia y a los Países Aliados". ${ }^{63}$ Sin embargo, al finalizar el conflicto armado, los Estados Unidos y Países Aliados volvieron a la producción de textiles y México, que para ese entonces enfrentaba un tipo de cambio sobrevaluado, sufrió una rápida caída de sus exportaciones manufactureras.

El resto de los años cuarenta y cincuenta fueron negativos para las empresas textiles porfirianas. Además de la caída en las exportaciones, que hizo que la producción real de textiles de algodón disminuyera en aproximadamente $11.5 \%$ entre $1946-47,{ }^{64}$ la competencia con nuevas fábricas, cuyos propietarios generalmente eran de origen árabe y judío, aumentó considerablemente. Al mismo tiempo, las fábricas porfirianas continuaban sin invertir en tecnología moderna. Después de 1945, muchos empresarios hicieron otro intento para modernizar sus fábricas. Prime-

${ }^{59}$ Secretaría de Hacienda y Crédito Público, Comisión de Aranceles, La Industria Textil del Algodón en México, op. cit., pp. 19-23.

${ }^{60}$ Durante la Convención, los representantes del trabajo propusieron establecer un salario mínimo de 4.50 pesos para toda la industria textil y los empresarios de 2.00 pesos. Finalmente se acordó un salario mínimo de 2.50 pesos, más cercano a lo que los empresarios habían sugerido.

${ }^{61}$ Entre 1940 y 1945 la producción textil creció en 173\% debido al aumento en la demanda externa. Ver Enrique Cárdenas, La Hacienda Pública y la política Económica en México, 1929-1958, 1994, México, El Colegio de México y Fondo de Cultura Económica, p. 126.

${ }^{62}$ Ibid., p. 96.

${ }^{63}$ Eduardo Oropeza, "Del Obraje de Contreras a la Fábrica de Hilados y Tejidos La Magdalena”, 2001, México, Tesis de Licenciatura, INAH, p. 146.

${ }^{64}$ Cárdenas, op. cit., Apéndice Estadístico, tabla A.6. 
ramente intentaron cambiar las restricciones legales. En ese año, el presidente de la Compañía Industrial Veracruzana (CIVSA) explicó en una junta del consejo de administración de la empresa "que era urgente para (la fábrica de) Santa Rosa, al igual que para la industria textil mexicana en general, modernizarse completamente [...] que era una cuestión de vida o muerte para la industria nacional". ${ }^{65}$ Sin embargo, sus esfuerzos fracasaron. "Solamente las nuevas fábricas establecidas después de la guerra estaban exentas de las restricciones impuestas por el contrato laboral de la industria" ${ }^{66}$ Además, los obreros continuaron obstruyendo los esfuerzos de modernización, incluso los que se llevaban a cabo en nuevas plantas. A pesar de que ellos manifestaban no estar en contra de la modernización, sus peticiones al gobierno mostraban lo contrario. Por ejemplo, en 1942, el sindicato de La Magdalena se unió con el de otras fábricas textiles en lo que llamaron "Pacto de Solidaridad y Ayuda Mutua" para discutir los problemas que los obreros confrontaban en las distintas fábricas. En 1953, esta unión sindical envió una carta al presidente Ruíz Cortines en donde, entre otros temas, mencionan que "ellos no estaban en contra de la modernización de las fábricas textiles mientras que los empresarios cumplieran con las leyes laborales y los contratos colectivos". ${ }^{67}$ El problema era que, además de la rígida lista de salarios, el contrato de trabajo del momento forzaba a la industria a mantener el mismo número de trabajadores empleados: "cualquier obrero que dejara su trabajo por cualquier razón tenía que ser reemplazado. Además, como (el contrato) establecía un sistema de ascenso basado en antigüedad, limitaba a las empresas a elegir y promover a los empleados con base en aptitud y esfuerzo". ${ }^{68}$ En la carta mencionada, la unión sindical solicitaba "la estandarización del sistema laboral en las fábricas modernas para que las no modernas puedan subsistir". ${ }^{69}$ En otras palabras, la unión estaba en contra de

${ }^{65}$ Gómez Galvarriato, op. cit., pp. 38-9.

${ }^{66}$ Banco Internacional de Reconstrucción y Desarrollo, The Economic Development of Mexico, 1953, Baltimore, p. 69.

${ }^{67}$ AGN, México, Presidentes: Ruiz Cortines Caja 432/135, junio de 1953.

${ }^{68}$ Segunda Ponencia de la Compañía Industrial de Orizaba S. A. en Primera Convención de Empresarios Textiles (Rama del Algodón), Memoria General, 1945, México, Servicio Nacional de Publicidad, p. 195.

${ }^{69}$ AGN, México, Presidentes: Ruiz Cortines Caja 432/135, junio de 1953. 
JOSÉ GALINDO

las excepciones en los contratos laborales de las que gozaban las fábricas modernas (básicamente el contrato era más flexible y permitía otro tipo de promociones laborales) porque esto generaría una mayor brecha entre fábricas textiles.

En 1953, el sindicato de La Magdalena, La Lucha, envió otra carta al presidente de la República, quejándose de que los dueños de la fábrica planeaban cerrarla por tiempo indefinido sin ningún compromiso con los trabajadores. También planeaban reducir a la mitad la jornada laboral y mantener plazas vacantes. El sindicato solicitaba al presidente que rechazara la propuesta hecha por los dueños a la Junta de Conciliación y Arbitraje, en donde los empresarios argumentaban que "las condiciones económicas de la empresa eran malas y que tenían que tomar medidas". De acuerdo con el sindicato, "habían habido despidos sin causa justa y sin compensación". También mencionan que "los dueños exageraban la mala situación de la industria textil para hacer todos estos cambios que les permitieran modernizar la fábrica". ${ }^{70}$

La Magdalena fue cerrada en marzo de 1954. De acuerdo con algunos reportes, lo que generó este cierre fue un conflicto artificial. En estos reportes, dos sindicatos de Puebla solicitaban al presidente la "rápida y justa resolución del conflicto artificial que llevó al cierre de la fábrica de algodón La Magdalena". ${ }^{71}$ La fábrica fue reabierta en 1954 y el sindicato La Lucha envió un muy afectuoso agradecimiento al presidente por la reapertura de la fábrica. En la carta, ellos ofrecen el más amplio apoyo al "gobierno que honorablemente presidía". También lo llamaban "un patriota". ${ }^{72}$ Después de 37 años, cuando este sindicato originalmente anarco-sindicalista fue creado, este hecho representaba un cambio notable en las relaciones entre los obreros y el Estado.

Parece ser que las medidas planeadas y/o efectuadas por los empresarios franceses, como cerrar la fábrica sin compensaciones laborales, reducir la jornada laboral y mantener plazas vacantes, eran medidas para adelgazar la fuerza de trabajo, lo que obligaría a los obreros a buscar otras fuentes de trabajo para modernizar la fábrica y volverse

${ }^{70}$ Ibid.. Leg. 1-6, noviembre de 1953.

${ }^{71}$ Ibid., 27 de marzo de 1954.

${ }^{72}$ Ibid., 31 de mayo de 1954. 
competitivos de nuevo al menos en el mercado interno. Incluso los obreros percibieron esto en las cartas que enviaron al presidente Ruíz Cortines. Sin embargo, la modernización jamás ocurriría. El sindicato La Lucha era lo suficientemente fuerte y tenía una muy buena relación con el gobierno.

A inicios de la década de los cincuenta, el $95 \%$ de los telares y el $85 \%$ de los husos que estaban en operación en México habían sido construidos antes de $1925 .^{73}$ Sin embargo, ya era tarde para modernizar la industria. Si desde principios de los cuarenta, como se mencionó, había quejas de los empresarios por la maquinaria que empleaban, para los cincuentas esta tecnología era absolutamente obsoleta. Además, para ese entonces, las fibras sintéticas estaban invadiendo el mercado mexicano. De hecho, después de la Segunda guerra mundial, corporaciones transnacionales productoras de fibras sintéticas como Celanese establecieron sucursales en México y empezaron a cambiar los patrones de consumo. Entre 1956 y 1965, la producción de fibras sintéticas pasó de 25,586 a 55,392 toneladas, lo que representó un incremento del $116.5 \%{ }^{74}$ A pesar de esto, La Magdalena todavía continuaría operando por algunos años. En 1973, un año antes de que la producción de fibras sintéticas sobrepasara la producción de fibras de algodón, La Magdalena cerró sus puertas. Al inicio de 1973, después de seis años de huelga, la Suprema Corte falló a favor de los obreros de la fábrica. La huelga había estallado básicamente por violaciones al contrato colectivo de trabajo. La compañía tuvo que pagar 47 millones de pesos para compensar a los trabajadores. Sin embargo, debido a que el pago no fue hecho, la fábrica fue expropiada y vendida a un precio muy bajo. Esta situación más que un problema, representó un alivio para los dueños de La Magdalena. Se pudieron deshacer de una fábrica no competitiva y vieja sin compensar a los trabajadores directamente. ${ }^{75}$

${ }^{73}$ Naciones Unidas, Departamento de Asuntos Económicos, Productividad de la Mano de Obra en la Industria Textil Algodonera de Cinco Países Latinoamericanos, 1951, Nueva York, p. 87.

${ }^{74}$ Cámara Nacional de la Industria Textil, Memoria Estadística, 1975, México, pp. 16-7.

${ }^{75}$ Oropeza, op. cit., pp. 149-53. 
JOSÉ GALINDO

\section{Conclusiones}

En este trabajo lo que se buscó fue demostrar dos cosas: por un lado, se analizó el desarrollo del movimiento obrero radical en la fábrica La Magdalena y se mostró que, a pesar de que los trabajadores de esta empresa fueron muy beligerantes entre finales del siglo XIX y el primer tercio del siglo XX, esta fábrica nunca dejó de estar dentro de las "top-ten" de la industria textil mexicana del algodón. Algunos argumentos expuestos, que jugaron un papel fundamental para explicar este primer resultado, fueron: la necesidad que tenían los obreros de conservar su trabajo; el control sobre los trabajadores en la fábrica por parte de los supervisores; el rechazo y combate del gobierno a las ideologías y organizaciones radicales; y la sobreproducción de las fábricas durante varios años del Porfiriato, la Revolución y los años veinte del siglo pasado.

El segundo objetivo del trabajo fue demostrar que los avances en la organización de los trabajadores y en materia laboral, que se desarrollaron de manera paralela al movimiento obrero radical de la fábrica La Magdalena en el primer tercio del siglo XX, llevaron a un arreglo institucional entre empresarios, obreros y gobierno que no ofreció incentivos a la transformación tecnológica y, como consecuencia, requirió de mayores tarifas para operar. ${ }^{76}$ Este patrón no cambió en las convenciones obrero-patronales subsiguientes a la de 1925-27, como la de 1937-39, a pesar de las propuestas de algunos industriales de introducir un esquema de salarios más flexible. El esquema de salarios introducido en la Convención Obrero Patronal de 1925-27, básicamente fijaba un número máximo de máquinas por trabajador y establecía salarios específicos por pieza producida. Bajo estas condiciones, los empresarios no tenían incentivos para introducir mejor tecnología porque no les permitiría reducir costos laborales, ya que los salarios por pieza tenían que mantenerse invariables. Además, en esa Convención también se crearon las "comisiones mixtas", que introdujeron el arbitraje en asuntos entre los obreros y patrones. Esto generó un gran obstáculo para el despido de los trabajadores en el futuro.

${ }^{76}$ Como observamos anteriormente, el incremento más representativo de las tarifas se dio entre 1926 y 1927. Sin embargo, después de este año, las tarifas se mantuvieron lo suficientemente altas para aislar a la industria nacional de la competencia externa. 
Todas estas “innovaciones" laborales, tuvieron consecuencias graves en la introducción de tecnología de punta en las fábricas textiles desde los años veinte. Como se explicó, a inicios de la década de los cincuenta, el 95\% de los telares y el $85 \%$ de los husos que estaban en operación en México habían sido construidos antes de 1925. De esta forma, después de mediados del siglo veinte, como se ilustró para el caso de La Magdalena, algunas empresas textiles comenzaron a cerrar sus puertas, lo que también fue en parte consecuencia de la introducción de fibras sintéticas al mercado nacional, cambiando los patrones de consumo. ${ }^{77}$

${ }^{77}$ El efecto de la introducción de fibras sintéticas en los patrones de consumo es un tema interesante para otra investigación. Aquí podemos mencionar que, entre 1956 y 1965, la producción de fibras sintéticas en México pasó de 25,586 a 55,392 toneladas, lo que representó un incremento del 116.5\%. 
La reproducción total o parcial de este artículo se podrá hacer si el ITAM otorga la autorización previamente por escrito. 